\title{
Patient-perceived empathy from nurses in Taiwan acute care settings
}

\author{
Sing-Ling Tsai ${ }^{1}$, Sinkuo Chai ${ }^{2}$, Hsiang Hwa Wang ${ }^{3}$ \\ ${ }^{1}$ Department of Nursing, Fu Jen Catholic University, New Taipei City, Taiwan \\ ${ }^{2}$ Department of Health Services Management, China Medical University, Taichung, Taiwan \\ ${ }^{3}$ Registered Nurse, Taipei Veterans General Hospital, Taipei, Taiwan \\ Email: sltsai001@gmail.com
}

Received 8 October 2013; revised 9 November 2013; accepted 23 November 2013

Copyright (C) 2013 Sing-Ling Tsai et al. This is an open access article distributed under the Creative Commons Attribution License, which permits unrestricted use, distribution, and reproduction in any medium, provided the original work is properly cited.

\section{ABSTRACT}

Purpose: The aim of this study was to understand patient-perceived empathy from nurse among different units of Taiwan acute care settings. Design: A crosssectional study was used with a questionnaire survey in acute care units and intensive care units of a medical center in northern Taiwan. Participants were selected by convenient sampling. A total of 548 patients at 14 acute care units and 4 intensive care units in a large Medical Center participated in this questionnaire survey. Methods: Scale of patient-perceived empathy from nurse including 15 item Likert scale was used in this study. The statistical software SPSS 15.0 for Windows was applied and Cronbach's $\alpha$, factor analysis, Pearson's correlation, $t$-test and one way ANOVA were conducted to analyze the data. Findings: Patients who were first-time hospitalization had higher scores of patient-perceived empathy from nurse than patients who have had hospitalizing experiences. The patient in surgical unit had the highest score of patient-perceived empathy from nurse. The empathy scale score of patient without ICU experience was significantly higher than patient with ICU experience. Also, patients without depressive history had higher score than patients with depression history. Conclusion: Patients with multiple admissions of hospitalization, ICU experience, and depressive history had lower score than other patients. It is suggested that the perception of patients with complicated disease or condition might be different. Thus, an in-depth caring knowledge of both emotions and disease was needed to support patients' perception of empathy. Clinical Relevance: The care of complicated patients especially with ICU experience needs strong knowledge based both on emotion and disease.
Keywords: Empathy; Patient Perception; Nurse; Scale; Acute Care Settings; Taiwan

\section{INTRODUCTION}

In a complex acute care of clinical setting under the short length of stay situation in Taiwan, it is important for nurses to use professional skills to enable patients to perceive their empathy and to guide patients in expressing their true feelings. Empathy is the perception of patients' feelings, namely, nursing personnel's understanding of their patients' feelings by placing themselves in their patients' position [1]. Education for the cultivation of nursing personnel has also affirmed that the use of empathetic attitudes and training for the application of relevant skills can improve communication with patients, enhance the nurse-patient relationship, and achieve the purpose of increasing the therapeutic effect on patients $[2,3]$.

It is the essential contribution of nursing profession in assessing and screening patient's emotional fluctuation through the nurse-patient relationship. Important clues of patient's depressed mood or even suicide attempt might emerge in the nurse-patient relationship and nurse could provide timely emotional care in collaborating with other professions [4].

However, there is limited knowledge of the patient's perception of empathy. Reynolds (2000) [5] developed Reynolds empathy measure (RES) to measure patient's perception of nurse's empathy through observing the verbal and nonverbal interaction between nurses and patients. RES was an interview scale, not a self-filling measurement. Mercer, Maxwell, Heaney and Watt (2004) [6] designed Consultation and Relational Empathy (CARE) to measure the patients' perception of consultant's attitude and behavior of empathy. It was used in the outpatient department for testing the patient-physician 
relationship. Kane, Gotto, Mangione, West and Hojat (2007) [7] used Jefferson Scale of Patient's Perceptions of Physician Empathy (JSPPPE) in the clinics of Thomas Jefferson University Hospital in measuring patients' perception of physicians' empathy. CARE and JSPPPE were used in outpatient settings only. In acute care settings, nurses not only needed to express their empathy, but also established nurse-patient relationship through the use of empathy and further conducted emotional assessment and care. Empathy is the essential step stone in taking care of patients.

Yu \& Kirk (2009) [8] emphasized user-centeredness being important in evaluating quality of empathy measurement tools. They reviewed 12 empathy measures, only two measures administered in the patient subjects. La Monica (1981) [9] developed empathy construct rating scale and administered it in patient subjects, but $\mathrm{Yu}$ and Kirk (2009) commented that patients' perspectives were not taken into account in developing the scale. Wheeler (1996) [10] developed perception of empathy inventory and administered it in patient subjects. Yu and Kirk (2009) commented that patients' views were not sought in its item generation.

Since empathy is the perception of patient's feelings by nurse, variables of nurse and patient are equally important to facilitate the expression and perception of empathy. Yu and Kirk (2008) [11] reviewed 29 papers of empathy and found that empathy was explored in relation to nurses' variables like age, experience, education, gender, attitudes, work place settings, and leadership style in 11 studies. Yet, we found variables of patients were seldom explored.

Wiseman (2007) [12] proposed that empathy is a process of nurse-patient relationship. Then, the variables of patient might contribute empathy as a process. WattWatson, et al. (2000) [13] assumed nurse's empathy as therapeutic factor in pain management and their study found that nurses' empathy did not significantly influence their patients' pain intensity or analgesia administered.

In addition to the above variables of patient perspectives, nurse's age, experience, education, gender, attitudes, work place settings, and leadership style, culture is another essential variable in the expression and perception of empathy. Most of the empathy studies were completed in the UK or North America. Cultural variations on empathy were not discussed, yet nurses work within a wide range of diverse cultural and language groups [14].

Study (Lee, Kuo, Tseng \& Turton, 2005) [15] in Taiwan has developed a caring behavior scale to measure the expression of caring behavior of student nurses. However, caring behavior is only a component of the expression of empathy. To date, there is limited knowledge of the empathy expressed by nurses in Taiwan acute care settings. Therefore, the purpose of this study was to understand patient-perceived empathy from nurse among different units of Taiwan acute care settings.

\section{RESEARCH METHOD}

The instrument used to measure patient-perceived empathy from nurses included fifteen items with Liker scale answers based on nursing experts' experience with reference to JSPPPE, CARE and RES. This instrument was applied in 2006 to 99 hospitalized patients of the chest department in a medical center in northern Taiwan after expert face validity being confirmed and factor analysis being performed.

\subsection{Research Design}

A cross-sectional study was used, and subjects were selected by convenient sampling. The subjects were hospitalized patients in internal medicine units, surgical units, gynecological units, and 4 intensive care units (ICU) in a medical center. A questionnaire survey was conducted.

\subsection{Research Tools}

This study used structured questionnaires to collect data. The contents of the questionnaires included basic information, a total of 15 items of the scale of patient-perceived empathy from nurses.

\subsubsection{Basic Information Questionnaire}

The basic information included subjects' demographic characteristics and disease status. The former included gender, date of birth, marital status, occupation, education level, religious belief, living status before hospitalization, family companionship during hospitalization, and frequency of family visits during hospitalization, among others. The latter included disease diagnoses, number of admissions, and the medical history of chronic diseases.

\subsubsection{Scale of Patient-Perceived Empathy from Nurses}

The scale which was developed by the authors based on ten to twenty years nursing experiences with reference to JSPPPE, CARE and RES included a total of 15 items. A Likert 5-point scale was used to obtain scores, ranging from "strongly agree" to "strongly disagree", denoted by 5 to 1 respectively. The patients were asked to choose the score according to their level of perception. Five nursing and sociology-related experts were invited to review the expert face validity, and the items of the scale were amended according to the experts' comments.

\subsection{Research Subjects and Study Sites}

The participants of this study were patients hospitalized 
in a medical center from 7 medical units, including neurology, nephrology, cardiology, oncology, metabolism, gastroenterology, chest units, and 7 surgical units including general surgery, neurosurgery, colorectal surgery, orthopedics, urologic surgery, cardiac surgery, gynecology, and 4 intensive care units (ICU). The inclusion criteria were: 1) patients who were hospitalized for more than three days; 2) conscious patients aged 20 and above who understood Chinese or Taiwanese; 3) patients without severe mental disorders and cognitive impairment; and 4) patients without visually or hearing impaired.

\subsection{Protection of Participants' Rights}

This study was approved by the institutional review board of a medical center. A total of five research assistants on the study team explained to the participants the research purpose, the procedures, and expected time needed for completion. Moreover, enrollment was not initiated until the participants' consent and signature on the informed consent document was obtained. Priority was given to the human rights of the participants, and the participants were entitled to agree or refuse to participate in the study. In addition, the participants were also allowed to withdraw from this study at any time. To protect their privacy, all of the questionnaires were coded anonymously. The research data were used for academic research only and were never disclosed.

\subsection{Data Collection}

The research assistants distributed the questionnaires to those participants who met the including criterion. If the participants could not complete the questionnaires by themselves due to aged, poor eyesight, illiterate, the research assistants would complete the questionnaires on their behalf by asking them the contents. The research assistants would read out the items of the questionnaires. If the participants could not hear the contents clearly or understand them, the research assistants would repeatedly read out the items, without providing any explanations. If the participants experienced any discomfort during the completion of the questionnaires, the research assistants would fully respect their request for discontinuance or termination of the questionnaires. The study team distributed 556 questionnaires from June 1, 2009 to October 31, 2009. A total of 548 effective questionnaires were returned, with a return rate of $98.6 \%$.

\subsection{Data Analysis}

After the data collection was completed, the statistical software SPSS 15.0 for Windows was used to perform reliability analysis on the scale. Cronbach's $\alpha$ was used to determine the internal consistency of the scale. Factor analysis was performed, and principal component analy- sis was used to extract the factors. After the orthogonal transformation was performed, the Eigen values, variance explained, total variance explained, and Cronbach's $\alpha$ of three factors were obtained. Pearson's correlation was used to understand the correlation among the variables. An independent sample $t$-test and one way ANOVA were used to analyze if there were any differences in the subjects' perceptions of empathy from nurses among subjects of different demographic characteristics and disease statuses.

\section{RESEARCH RESULTS}

\subsection{Demographic Data of the Subjects}

According to the 548 returned questionnaires, 128 of the participants $(23.4 \%)$ were hospitalized in ICUs, while 420 were hospitalized in general units. There were 30 participants from each of 14 units enrolled, for a total of 420 participants $(77 \%)$. As for gender, 348 of the participants were male $(63.5 \%)$, and 200 were female $(36.5 \%)$. As for marital status, 415 of the participants were married (75.7\%), 67 unmarried participants (12.2\%), and the rest were divorced, separated, or widowed. As for the age distribution, mean age was 62.95 years old, ranged from 20.77 to 96.60 , half of the participants were over the age of 65 . As for occupation, most of the participants were unemployed $(63.7 \%)$, while the rest were engaged in public service, industry, commerce, or other occupations (all together less than 9\%). As for education level, $32 \%$ of the subjects were junior college graduates or above, $26 \%$ were senior high school graduates, and $42 \%$ were elementary school graduates or under or illiterate. As for religious beliefs, approximately $50 \%$ of the subjects had religious beliefs but seldom participated in religious activities. As for living method, most of the participants lived with their families $(90 \%)$, while the rest lived alone or in long-term care facilities. During hospitalization, approximately $8 \%$ of the participants were not looked after by any family members, $8 \%$ were looked after by foreign domestic helpers, and the rest of the participants were looked after by families and friends. As for visits by family and friends during hospitalization, approximately $80 \%$ of the participants were frequently visited by families and friends. As for the number of admissions, $45 \%$ of the participants had been hospitalized more than four times, $16 \%$ were hospitalized for the first time, $16 \%$ were hospitalized for the third time, and the rest of the participants were hospitalized for the second time. As for the medical history of chronic diseases, 58\% of the subjects suffered from chronic diseases.

\subsection{Reliability and Validity of the Instrument}

The Cronbach's $\alpha$ of the scale of patient-perceived em- 
pathy from nurses was 0.89 indicating good internal consistency of this instrument.

Principal component analysis was used to extract factors and obtain the Varimax after orthogonal transformation was performed. Three factors were extracted based on Eigen value $\geq 1$ and were named "nurses' expressions", "patients' feedback", and "patients' expectations", as shown in Table 1. The Eigen values of the three factors were $6.49,1.78$, and 1.29 , respectively. The total variance explained was $63.71 \%$. The Cronbach's $\alpha$ were $0.90,0.79$, and 0.80 , respectively. The mean scores of nurses' expressions, patients' expectations, and patients' feedback were 4.04, 4.31, and 3.81, respectively, as shown in Table 2.

\subsection{Correlations between the Empathy Scale Scores and Demographic and Disease Variables}

The empathy scale scores were no statistic different between gender and above and under age of 65, among marital status, occupation, education level, religious belief, living status before hospitalization, family companionship during hospitalization, and frequency of family visits during hospitalization, disease diagnoses, and the medical history of chronic diseases. But there were differences among ICU, medical and surgical ward ( $p=$ $0.023)$. The patient in surgical ward had the highest score (61.33). The empathy scale score of patient without ICU experience was significantly higher than patient with ICU experience $(p=0.004)$. Patients who were first time hospitalization had higher scores than patients who have had hospitalizing experience $(p=0.037)$. Also, patients without depressive history had higher score than patients with depression history $(p=0.041)$ in Table 3 .

\section{DISCUSSION}

Empathy is the ability to understand the experiences, behavior, and feelings of other people, and the ability to enable others to perceive that they are understood. In the current busy clinical medical environment of Taiwan, empathy plays an important role in both doctor-patient relationships and nurse-patient relationships. If medical and nursing personnel can express empathy and understand patients' inward status, feelings, and needs from the patients' perspective, it is believed that both care quality and patient satisfaction can be improved. Burhans and Alligood (2010) [16] also found that empathy was one essence of quality nursing care.

Table 1. Factor analysis on the scale of patient-perceived empathy from nurses $(N=548)$.

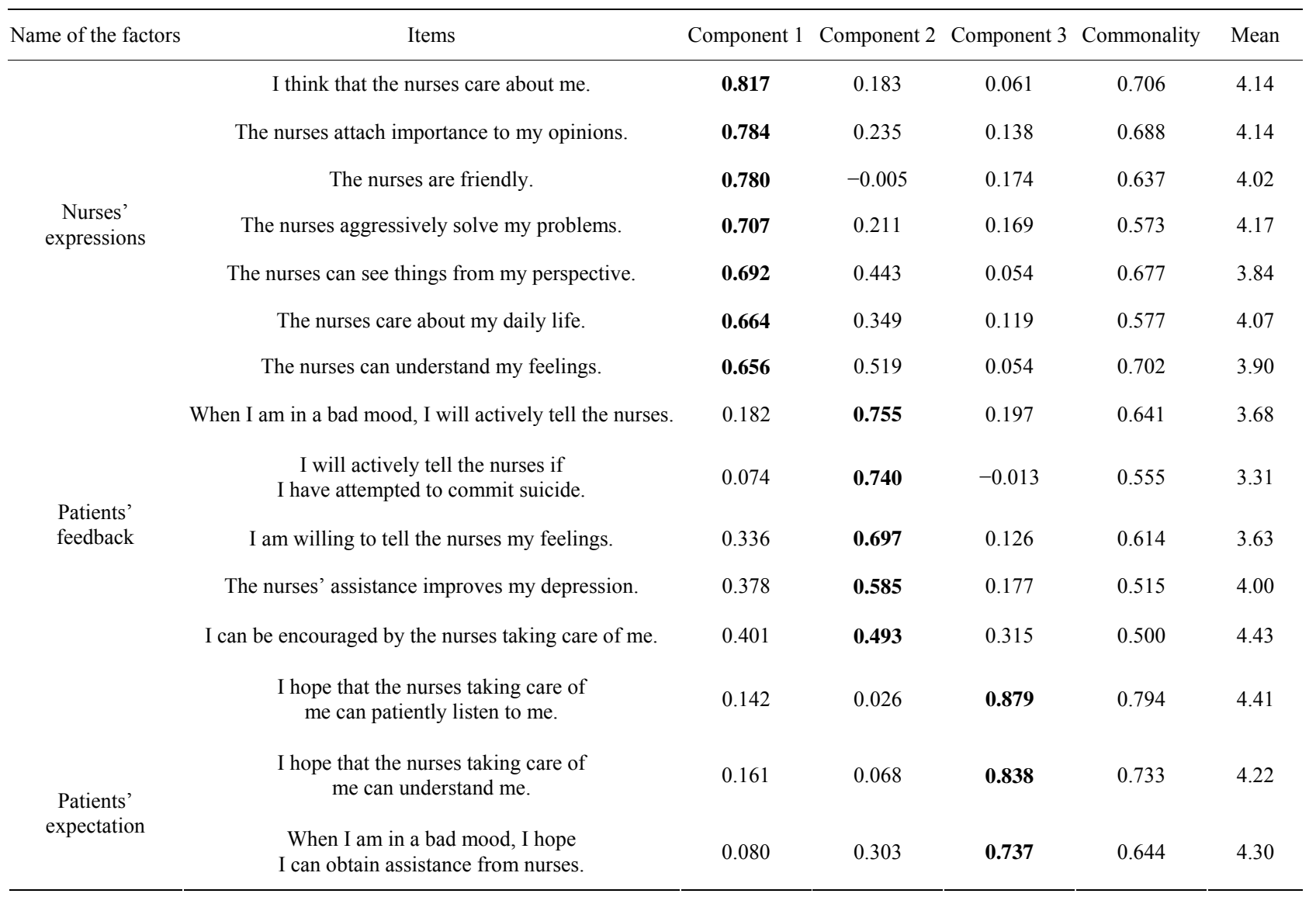


Table 2. Summary of analysis on the scale of patient-perceived empathy from nurses.

\begin{tabular}{ccccccc}
\hline Name of factors & Number of items & Mean score & Eigen value & Variance explained & Total variance explained & Cronbach's $\alpha$ \\
\hline Nurses' expression & 7 & 4.04 & 6.488 & 43.251 & 43.251 & 0.900 \\
Patients' feedback & 5 & 3.81 & 1.777 & 11.849 & 55.099 & 0.786 \\
Patients' expectation & 3 & 4.31 & 1.291 & 8.606 & 63.705 & 0.799 \\
\hline
\end{tabular}

Table 3. Correlation between disease variables and the scale of patient-perceived empathy from nurses $(N=548)$.

\begin{tabular}{|c|c|c|c|c|c|}
\hline \multirow{2}{*}{ Variable } & \multirow{2}{*}{$n$} & \multicolumn{4}{|c|}{ The scale of patient-perceived empathy from nurses } \\
\hline & & $M e a n \pm S D$ & $t / F$ value & $\boldsymbol{P}$ value & LSD test \\
\hline \multicolumn{6}{|l|}{ Unit } \\
\hline Internal medicine & 210 & $60.32 \pm 7.27$ & \multirow{3}{*}{3.789} & \multirow{3}{*}{$0.023^{*}$} & \multirow{6}{*}{ (2) $>$ (3) } \\
\hline Surgical & 210 & $61.33 \pm 7.61$ & & & \\
\hline Intensive Care Unit (ICU) & 128 & $59.11 \pm 6.45$ & & & \\
\hline ICN experience in hospitalization & & & & & \\
\hline No & 379 & $61.01 \pm 7.33$ & \multirow{2}{*}{2.893} & \multirow{2}{*}{$0.004^{*}$} & \\
\hline Yes & 169 & $59.09 \pm 6.93$ & & & \\
\hline \multicolumn{6}{|l|}{ Relatives or friends visiting during hospitalization } \\
\hline No & 14 & $58.29 \pm 5.58$ & \multirow{4}{*}{2.192} & \multirow{4}{*}{0.088} & \\
\hline Yes, always & 438 & $60.80 \pm 7.24$ & & & \\
\hline Yes, often & 83 & $59.24 \pm 7.14$ & & & \\
\hline Yes, seldom & 13 & $57.62 \pm 8.99$ & & & \\
\hline \multicolumn{6}{|l|}{$\begin{array}{c}\text { Relatives or friends accompanied during } \\
\text { hospitalization }\end{array}$} \\
\hline No & 41 & $58.93 \pm 6.50$ & \multirow{3}{*}{.972} & \multirow{3}{*}{0.379} & \\
\hline Yes, accompanied by relatives or friends & 462 & $60.57 \pm 7.35$ & & & \\
\hline Yes, accompanied by a foreign helper & 45 & $60.29 \pm 6.92$ & & & \\
\hline \multicolumn{6}{|l|}{ Times of admission } \\
\hline First time & 89 & $61.89 \pm 7.42$ & \multirow{2}{*}{2.085} & \multirow{2}{*}{$0.037^{*}$} & \\
\hline More than twice & 459 & $60.14 \pm 7.20$ & & & \\
\hline \multicolumn{6}{|l|}{ History of chronic illness } \\
\hline No & 229 & $60.75 \pm 7.28$ & \multirow{2}{*}{-0.895} & \multirow{2}{*}{0.371} & \\
\hline Yes & 319 & $60.19 \pm 7.25$ & & & \\
\hline \multicolumn{6}{|l|}{ History of depression } \\
\hline No & 537 & $60.51 \pm 7.19$ & \multirow{2}{*}{2.047} & \multirow{2}{*}{$0.041^{*}$} & \\
\hline Yes & 11 & $56.00 \pm 9.64$ & & & \\
\hline
\end{tabular}

The findings in this study were based on patient's perception. The average score of nurses' expressions was 4.04 in a 5 point scale, which showed that patients appraised nurse's expression of empathy including care, friendly, understanding feelings, seeing patient's perspective, and actively solving patient's problem.
The average score of the patients' feedback factor was 3.81. The mean score of patients' feedback consisted of willingness to tell nurse about their feelings, actively telling bad mood, attempt of suicide were 3.63, 3.68 and 3.31 respectively. In today's modern Taiwanese culture, people would not tell others about their feeling except 
the familiar friend or family, especially when they were in bad mood. Thus, patient's willingness to tell his/her feelings was based on a familiar nurse-patient interpersonal relationship. In this study, 72.6\% - 73.6\% (3.63/5 $3.68 / 5$ ) patients agreed to tell their feeling or bad mood. This data reflected the nurse-patient relationships being fairly reasonable.

Furthermore, $66.2 \%$ patients would actively to tell their nurse about their suicide attempt. Nurses could grasp this opportunity to screen the possibility of suicide and made further referral to the expert team. Yet, there were $33.8 \%$ not agreeing in telling their nurses about their suicide attempt in spite of $80 \%-88 \%(4.43 / 5-4 / 5)$ agreeing nurse's encouragement and assistance in improving patients' depression. Thus, patient's willingness to tell their feelings challenged nurse's interpersonal skill in the nurse-patient relationship. Also, time is important in expression empathy of active listening and patience. A heavy clinical nursing workload may have impeded nurses from having sufficient time to have an in-depth understanding of their patients' feelings. It took time when nurse dealing with patient in bad mood or depressive state which happened a lot in the acute care settings. Webster and Bryan (2009) [17] also strengthened the important of staff sufficient time for older patient's perception of dignity.

Teng and his colleagues (2010) [18] study found that nurse-perceived time pressure did not have negative association with patient-perceived empathy. Teng's measurement of empathy was a subscale and only included individual attention, in a caring fashion, had best interest at heart, understood needs. The scale in this study not only embraced patient's perception of nurse's expression of empathy, but also included patient's feedback of actively telling their feelings or bad mood, even attempt of suicide and patient's hope of assistance in bad mood. Thus, time is important for patients to express their feelings as interacting with nurses' empathy.

Patient's expectation which included understanding, patiently listening, and obtaining assistance from nurses when in bad mood was high in the average score of 4.31 . This result demonstrated that patient had high expectation from nurse in terms of empathy. Thus, nurses had the chance to express their empathy in a stable nursepatient relationship and could assess patient's mood.

The results showed that the units (internal medicine unit, surgical unit, and ICU), previous experiences of being admitted to an ICU, the number of admissions, and a medical history of depression significantly affected the score of patient-perceived empathy from nurses. Among them, the empathy score of the patients hospitalized in surgical unitts was the highest, and the reason might be that patients at surgical units mainly suffered from physical pain after surgery, and it was clear to observe, iden- tify, and solve such problems. In interacting with nurses, the surgical patients had higher perceived-empathy score.

In terms of the number of admissions, the score of the patient-perceived empathy of patients who were hospitalized for the first time was higher than that of those who were hospitalized for more than two times. The reason might be that the diseases of those who were hospitalized for more than twice were more complicated. Thus, good attitude is not enough, an in-depth caring knowledge is needed to support the perception of empathy.

The condition of the patients hospitalized in the ICU was severe, or they had experienced discomfort caused by treatment behavior (e.g. intubation, protection constraints, etc.), and because the work of the nurse was more complicated, the empathy score of such patients was lower. The score of the patients who had not been admitted to an ICU during hospitalization was higher than that of those who had been admitted to one. The reason might be that ICU patients had experienced severe diseases, complicated treatment and needed more attention in terms of empathy.

Stayt (2009) [19] conducted interviews with 12 nurses working in ICU and found the significance of death, breaking bad news and interpersonal relationships are sources of emotional stress for the critical care nurse caring for the patients and their family of the critically ill. From these themes, there were lots of emotional demands for patients walking through death and were sources of emotional stress for nurses. Thus, these serious and complicate situations in ICU challenged patient perceived-empathy.

The score of the patient-perceived empathy of patients without a medical history of depression was higher than those with it. The patients with depression had a higher demand for empathy, and they might have failed to adequately express their feelings since the illness affected their interpersonal interactions. As a result, the score was lower.

There were several limitations of this study. This study did not measure the impact of severity of diseases and the workload of nurses on the score of empathy expressions. Also, nurses' variables which might influence their expression of empathy were not measured. Future studies may further investigate how patient's disease severity, nurse's variables and workload affect patient-perceived empathy and nurses' expressions of empathy.

\section{CONCLUSIONS}

Empathy was an essence of quality nursing care as indicated by Burhans and Alligood (2010). If nurses could express empathy and understand patients' inward status, feelings, and needs from the patients' perspective, the 
care quality and patient satisfaction could be improved.

Patients perceived nurses having good expression of empathy. However, patient's expectation of empathy was even higher as shown by the mean score of 4.31. They appraised the encouragement and assistance in improving their depression mood, nevertheless they hesitated to tell nurse about their feelings or bad mood. This is a challenge for nurses especially working in a busy medical environment.

The multiple time hospitalization patient, patient with ICU experience and with depressive history had lower score than other patients. It is suggested that the perception of patients with complicated disease or condition might be different. Thus, an in-depth caring knowledge of both emotions and disease was needed to support patients' perception of empathy.

\section{REFERENCES}

[1] Alligood, M.R. (2005) Rethinking empathy in nursing education: Shifting to a developmental view. Annual Review of Nursing Education, 3, 299-309.

[2] Ancel, G. (2006) Developing empathy in nurses: An inservice training program. Archives of Psychiatric Nursing, 20, 249-257. http://dx.doi.org/10.1016/j.apnu.2006.05.002

[3] Halpern, J. (2007) Empathy and patient-physician conflicts. Journal of General Internal Medicine, 22, 696-700. http://dx.doi.org/10.1007/s11606-006-0102-3

[4] Taur, F.M., Chai, S.K. and Tsai, S.L. (2012) Evaluating the suicide risk screening scale used by general nurses on patients with chronic obstructive pulmonary disease and lung cancer: A questionnaire survey. Journal of Clinical Nursing, 21, 398-407. http://dx.doi.org/10.1111/j.1365-2702.2011.03808.x

[5] Reynolds, W., Scott, P.A. and Austin, W. (2000) Nursing, empathy and perception of the moral. Journal of $A d$ vanced Nursing, 32, 235-242. http://dx.doi.org/10.1046/j.1365-2648.2000.01440.x

[6] Mercer, S.W., Maxwell, M., Heaney, D. and Watt, G.C. (2004) The consultation and relational empathy (CARE) measure: Development and preliminary validation and reliability of an empathy-based consultation process measure. Family Practice, 21, 699-705. http://dx.doi.org/10.1093/fampra/cmh621

[7] Kane, G.C., Gotto, J.L., Mangione, S., West, S. and Hojat, M. (2007) Jefferson scale of patient's perceptions of physician empathy: Preliminary psychometric data. Croatian Medical Journal, 48, 81-86.
[8] Yu, J. and Kirk, M. (2009) Evaluation of empathy measurement tools in nursing: Systematic review. Journal of Advanced Nursing, 65, 1790-1806. http://dx.doi.org/10.1111/j.1365-2648.2009.05071.x

[9] La Monica, E.L. (1981) Construct validity of an empathy instrument. Research in Nursing and Health, 4, 389-400. http://dx.doi.org/10.1002/nur.4770040406

[10] Wheeler, K., Marrett, E.A.M. and Lahey, E.M. (1996) A study of empathy as a nursing care outcome measure. International Journal of Psychiatric Nursing Research, 3, 281-289.

[11] Yu, J. and Kirk, M. (2008) Measurement of empathy in nursing research: Systematic review. Journal of Advanced Nursing, 64, 440-454. http://dx.doi.org/10.1111/j.1365-2648.2008.04831.x

[12] Wiseman, T. (2007) Toward a holistic conceptualization of empathy for nursing practice. Advances in Nursing Science, 30, E61-E72. http://dx.doi.org/10.1097/01.ANS.0000286630.00011.e3

[13] Watt-Watson, J., Garfinkel, P., Gallop, R., Stevens, B. and Streiner, D. (2000) The impact of nurses' empathic responses on patients' pain management in acute care. Nursing Research, 49, 191-200. http://dx.doi.org/10.1097/00006199-200007000-00002

[14] Brunero, S., Lamont, S. and Coates, M. (2010) A review of empathy education in nursing. Nursing Inquiry, 17, 64-73. http://dx.doi.org/10.1111/j.1440-1800.2009.00482.x

[15] Lee, H.J., Kuo, C.L., Tseng, H.F. and Turton, M.A. (2005) Development of an instrument to measure caring behaviors in nursing students in Taiwan. International Journal of Nursing Studies, 42, 579-588. http://dx.doi.org/10.1016/j.ijnurstu.2004.09.018

[16] Burhans, L.M. and Alligood, M.R. (2010) Quality nursing care in the words of nurses. Journal of Advanced Nursing, 66, 1689-1697. http://dx.doi.org/10.1111/j.1365-2648.2010.05344.x

[17] Webster, C. and Bryan, K. (2009) Older people's views of dignity and how it can be promoted in a hospital environment. Journal of Clinical Nursing, 18, 1784-1792. http://dx.doi.org/10.1111/j.1365-2702.2008.02674.x

[18] Teng, C.-I., Hsiao, F.-J. and Chou, T.-A. (2010) Nurseperceived time pressure and patient-perceived care quality. Journal of Nursing Management, 18, 275-284. http://dx.doi.org/10.1111/j.1365-2834.2010.01073.x

[19] Stayt, L.C. (2009) Death, empathy and self preservation: The emotional labour of caring for families of the critically ill in adult intensive care. Journal of Clinical Nursing, 18, 1267-1275. http://dx.doi.org/10.1111/j.1365-2702.2008.02712.x 